\title{
Perceptual integration of tertiary taste mixtures
}

\author{
ROBERT L. MCBRIDE \\ Sensory Research Centre, CSIRO Division of Food Processing, Sydney, Australia \\ and \\ DAVID C. FINLAY \\ University of Newcastle, Newcastle, Australia
}

\begin{abstract}
Integration psychophysics was used to explore the taste perception of mixtures of sucrose, fructose, and citric acid. Three levels of each stimulus were varied in a $3 \times 3 \times 3$ factorial design. Subjects rated total intensity, sweetness, and acidity of the 27 mixtures on graphic rating scales. Consistent with earlier work, the perceived total intensity of the tertiary mixtures was found to be dictated by the intensity of the (subjectively) stronger component alone (i.e., either the integrated sweetness or the acidity, whichever was the more intense). In contrast, the sweetness and acidity of the mixture were susceptible to mutual suppression: Sweetness suppressed acidity, acidity suppressed sweetness. There was, however, a difference between sucrose and fructose in their interactions with citric acid, fructose being the more susceptible to suppression. This selectivity of suppression indicates that the two sweetnesses could not have been inextricably integrated. Implications for taste coding are discussed, and the findings are reconciled in terms of two separate coding mechanisms: one for taste intensity, another for taste quality.
\end{abstract}

Over recent years, there has been some resurgence of interest in the perception of taste mixtures-both homogeneous and heterogeneous (Frijters, 1987; Laing, Cain, McBride, \& Ache, 1989; McBride \& Anderson, in press). Homogeneous mixtures are those in which the components evoke an apparently unitary taste sensation: For example, the taste of honey is a homogeneous mixture, its apparently unitary sweetness evoked by two sugars, fructose and glucose. The taste of lemonade, on the other hand, consists of two distinct sensations, sweetness and acidity (sourness), and it is therefore a heterogeneous mixture. In reality, most foods and drinks are heterogeneous taste mixtures, so an understanding of their perception is of no small practical importance.

A pattern is beginning to emerge for the perceptual integration of homogeneous taste mixtures, at least for binary mixtures (De Graaf \& Frijters, 1988; McBride, 1982, 1986, 1988, 1989, unpublished data). It appears that the sweetnesses of binary sugar mixtures are nearadditive at low concentrations, but that they fall increasingly short of additivity as the concentrations grow.

For (binary) heterogeneous mixtures, however, the patterns are quite different. First, it must be realized that heterogeneous mixtures evoke three separate sensations: the total (overall) intensity of the mixture, irrespective of taste quality; the intensity of the first component; and the intensity of the second component. According to recent studies on mixtures of sucrose and citric acid (Frank

We thank Sally Dewis and Kerry Easton for their technical support This research was supported by Grant 32/280/001 from the CSIRO/ University of Newcastle Collaborative Research Fund. Correspondence may be addressed to Robert L. McBride, Sensometrics Pty. Lid., 2/357 Military Road, Mosman, NSW 2088, Australia.
\& Archambo, 1986; McBride, 1989; McBride \& Finlay, in press), total intensity is determined by the intensity of the stronger, or dominant component alone; there is apparently no integration of the two components to produce total intensity. This effect, termed the dominant component model, has also been observed in a recent neurophysiological study of taste mixtures (Smith, 1989). In contrast, the tastes of the individual components (i.e., sweetness and acidity), clearly exhibited an interaction, manifest as mutual suppression.

This paper extends the research to the perception of tertiary taste mixtures; specifically, to mixtures of sucrose, fructose, and citric acid. This tertiary mixture, although heterogeneous, does contain within it the binary homogeneous mixture sucrose/fructose. Study of this "mixture within a mixture" may help elucidate the locus of stimulus integration. For example, does an integration of sweetness occur first, with the total sweetness then interacting with the acid? Or does the acid interact individually with each sugar? Will the dominant component model hold for a tertiary mixture?

\section{METHOD}

\section{Subjects}

Thirty subjects-staff and students from the Department of Psychology, University of Newcastle-participated voluntarily. None had had any experience in sensory testing.

\section{Stimuli}

The stimuli were mixtures of sucrose, fructose, and citric acid (all reagent grade) dissolved in distilled water. Three levels of sucrose, $0,0.172$, and $0.800 \mathrm{M}$, three levels of fructose, $0,0.259$, and $1.200 \mathrm{M}$, and three levels of citric acid, $0,0.006$, and $0.050 \mathrm{M}$, were combined factorially, giving $27 \mathrm{stimuli}$ in all. Thus, the weakest stimulus consisted of distilled water only; the strongest stimulus 
consisted of 0.800 moles of sucrose and 1.200 moles of fructose and 0.050 moles of citric acid in 1 liter of distilled water. The concentrations were chosen on the basis of previous work (McBride, 1987, 1989).

Solutions were made up at least $24 \mathrm{~h}$ before testing and stored at $5^{\circ} \mathrm{C}$. At evaluation, each sample consisted of $10-\mathrm{ml}$ of solution served at ambient temperature $\left(20^{\circ} \mathrm{C}\right)$ in a small polystyrene cup.

\section{Response Scales}

The subjects made their responses in a booklet of response sheets, one sheet per stimulus. Each sheet contained three $150-\mathrm{mm}$ graphic rating scales, one each for the assessment of total intensity, sweetness, and acidity. For total intensity, the descriptors "No taste at all," "Moderate," and "Extremely strong"' were positioned 0, 75, and $150 \mathrm{~mm}$, respectively, from the left-hand end of the scale. The corresponding descriptors for sweetness were "No sweetness at all," "Moderately sweet," and "Extremely sweet"; for acidity, they were "No acid at all," "Moderately acid," and "Extremely acid."

\section{Procedure}

The subjects assessed all stimuli at each of two separate sessions (replicates) held a few days apart, providing a total of 60 observations per stimulus. At the commencement of each session, the subjects tasted the strongest mixture $(0.800 \mathrm{M}$ sucrose/1.200 M fructose $/ 0.050 \mathrm{M}$ citric acid) as a guide to the sensory range. They were then required to "sip and spit" each stimulus according to the (random) order specified in the response booklet, and to rate the total intensity (sweetness, sourness) on the appropriate graphic rating scale. Rinsing with distilled water and a $30-\mathrm{sec}$ break were mandatory between stimuli.

The three response sets were analyzed with separate analyses of variance.

\section{RESULTS AND DISCUSSION}

\section{Perception of Total Intensity}

Mean ratings for total intensity are given in the three factorial plots in Figure 1. Sucrose concentration is given on the abscissa (log scale), the curves correspond to levels of citric acid, and the separate panels to different fructose levels. For the purpose of quantitative comparisons, Figure 1 also contains the least significant difference $(L S D)$ : Any two data points that are separated vertically by more than one $L S D$ are significantly different $(p<.05)$.

Figure 1A is essentially a factorial plot of sucrose/citric mixtures, fructose being at zero. It follows the same "dominant component" pattern as has previously been established (Frank \& Archambo, 1986; McBride, 1989; McBride \& Finlay, 1989; McBride \& Johnson, 1987).

Consider the mean rating for the total intensity of the highest level of citric acid alone (citric 2; top data point on the ordinate). When increasing concentrations of sucrose are added to citric 2, that is, moving rightward along the curve, there is no increase in total intensity; even the addition of high $(0.800 \mathrm{M})$ sucrose concentration does nothing to augment the total intensity of the mixture.

The middle concentration of citric acid (citric 1) likewise dictates the total intensity of the mixture, until the sucrose is added at its highest level (sucrose 2); at this point, the total intensity is virtually the same as the total intensity of sucrose 2 when tasted alone (bottom curve). In this case, the sucrose alone dictates the intensity of the mixture.
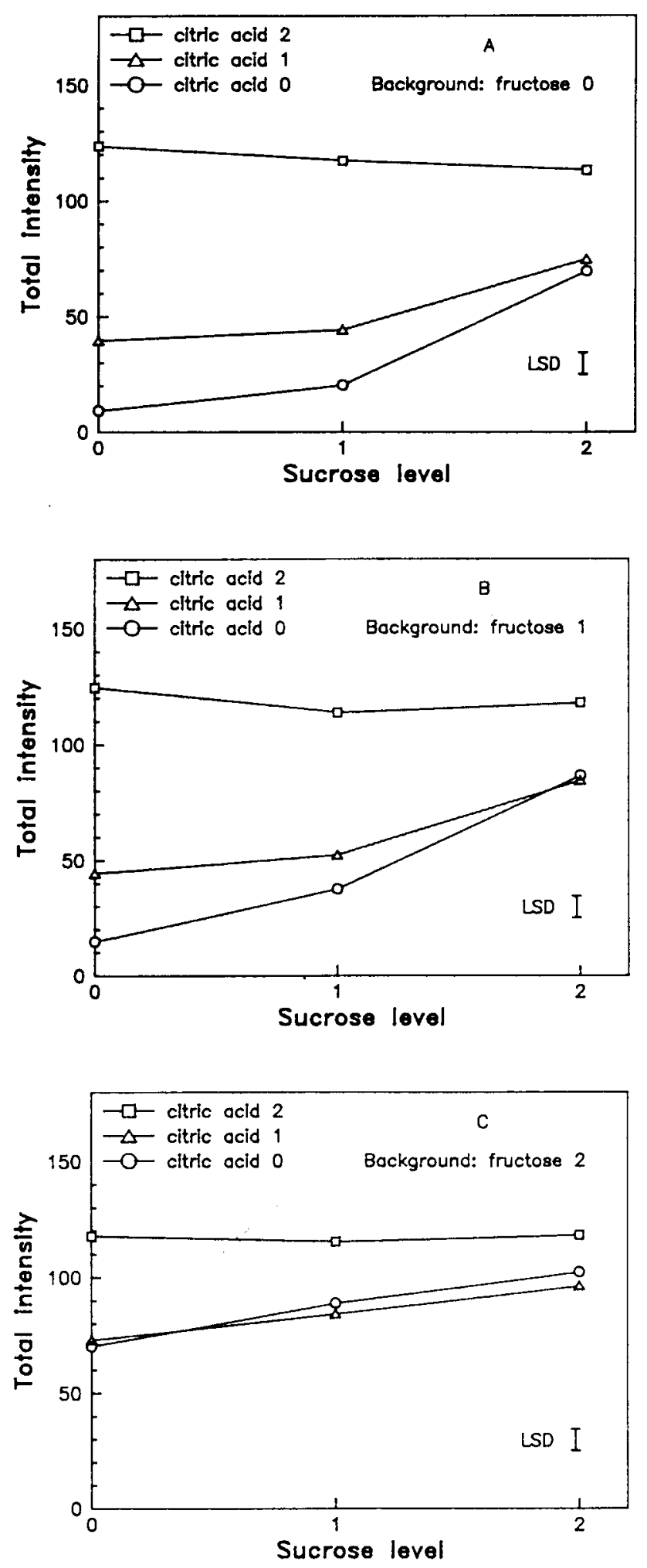

Figure 1. Mean ratings for the total intensity of the 27 sucrose/fructose/citric acid mixtures. Sucrose concentration is given on the (log) abscissa; each curve represents a different level of citric acid; and each panel represents a different level of fructose. Within each panel, data points separated (vertically) by more than one $L S D$ are significantly different $(p<.05)$. 
Inclusion of a second sugar at a medium level (fructose 1; Figure 1B) makes for three-component mixtures, but it has little effect on the response pattern. Citric 2 still dictates the total intensity of its mixtures; and citric 1 dictates the intensity of the citric $1 /$ sucrose 1 /fructose $1 \mathrm{mix}$ ture. However, the sweetness of sucrose 2 /fructose 1 dictates the total intensity of the citric $1 /$ sucrose 2 /fructose 1 mixture. That is, the integrated sweetness determines total intensity, not the sweeter of the individual sugars. This integration of sweetness intensities is also evidenced by an increase in ratings in the bottom curve of Figure 1B (i.e., compared with the bottom curve in Figure 1A).

Inclusion of the second sugar at a high level (Figure 1C) changes the pattern. Citric 2 still dictates the intensity of its mixtures (top curve). But the total intensity of fructose 2 alone (bottom data point on the ordinate) now exceeds the total intensity of citric 1 alone (middle data point on the ordinate in Figure 1A). Consequently, it is now the sugars that dictate total intensity: The two bottom curves are almost coincident, indicating that the addition of citric 1 had no effect.

Note that the two bottom curves in Figure 1C are not parallel to the abscissa. As total concentration of sugars increases, so does total intensity. Again, this confirms an integration of sweetness. This is shown more clearly in Figure 2, which gives the $3 \times 3$ factorial data plot for the sugars alone (i.e., with citric at zero). The pattern is very similar to those previously obtained for sucrose/fructose mixtures (De Graaf \& Frijters, 1988; McBride, 1982, 1986).

It should be mentioned that the horizontality of the top curve in Figure 1 is not a scaling artifact (i.e., a ceiling effect). Although it is not readily apparent from these $3 \times 3$ plots, previous work with more elaborate $(5 \times 5)$ factorial designs has clearly demonstrated that the effect also $\alpha$ curs at lower intensity levels (McBride, 1989; McBride \& Finlay, 1989).

In summary, then, these three-component mixtures support the dominant component model for the perception

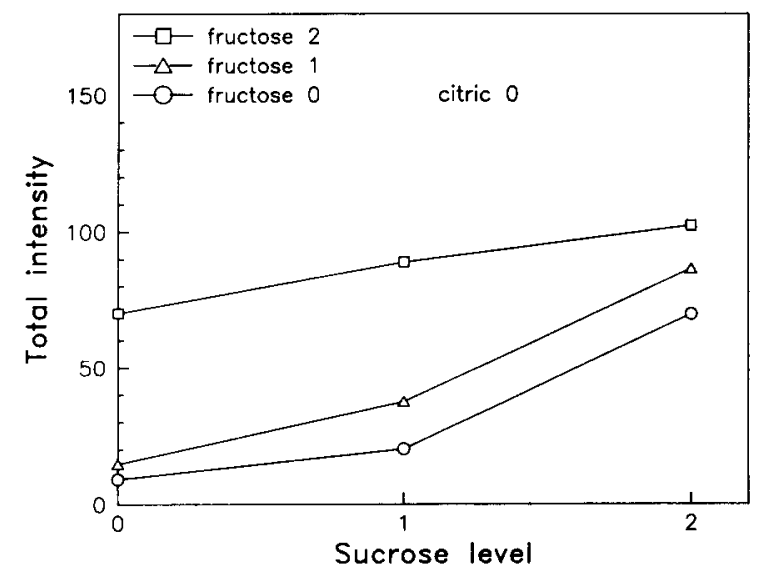

Figure 2. Mean total intensity ratings for the nine sucrose/fructose mixtures (i.e., citric acid at zero). Sucrose concentration is given on the (log) abscissa, and each curve represents a different level of fructose. of total intensity. Insofar as the judgment of total intensity is concerned, it appears that the individual sweetnesses are integrated and that they thereafter act as a single component.

\section{Sweetness}

The mean sweetness responses are given in Figure 3, together with the $L S D$. The relevant data points in Figures $3 \mathrm{~A}-3 \mathrm{C}$ confirm that the sweetness of sucrose 1 alone was equivalent to the sweetness of fructose 1 alone, similarly for sucrose 2 and fructose 2 .

According to Figure 3A, citric acid, even at high concentration, had virtually no suppressive effect on the sweetness of sucrose. This is consistent with earlier findings (Frank \& Archambo, 1986; McBride, 1989; McBride \& Finlay, 1989; McBride \& Johnson, 1987) that only very high concentrations of citric acid can suppress the sweetness of sucrose.

For fructose, however, Figure $3 \mathrm{C}$ shows a different picture. The data points on the ordinate indicate that citric acid had a marked suppressive effect on the sweetness of fructose alone. So, even though they were of equal sweetness, the two sugars differed in their interaction with citric acid, the sweetness of fructose being far more susceptible to suppression. This makes for a somewhat paradoxical effect: Adding more fructose to a mixture of sucrose/ fructose/citric acid does not necessarily increase the overall sweetness, because the sweetness of the fructose is suppressed by the citric acid. For instance, sucrose 2/ fructose $2 /$ citric 2 (Figure 3C) is no sweeter than sucrose $2 /$ fructose $1 /$ citric 2 (Figure 3B).

This finding has practical significance. On a weight basis, fructose is slightly sweeter than sucrose, and it should, logically, be a more effective sweetener. But if fructose is to be mixed with citric acid, a common constituent of many foods and drinks, the sweetening advantage would be lost.

\section{Acidity}

Figure 4 gives the mean acidity response and their $L S D$. Just as, in Figure 3 above, the two sugars were differntially suppressed by citric acid, so the acidity of citric acid was differentially suppressed by the two sugars. Sucrose suppressed acidity more effectively. For example, fructose 2 suppressed the acidity rating of citric 2 from 120 to 101 (Figure 4A), whereas sucrose 2 suppressed it to 93 (cf. top curves in Figures 4A and 4C). This also held in the tertiary mixtures. Sucrose 1 /fructose 2 , which was equivalent in sweetness to sucrose 2 /fructose 1 (ratings of 98 and 96, respectively, in Figures $3 B$ and $3 C$ ), reduced the acidity of citric 2 from 120 to 92 (Figure 4B), whereas sucrose 2 /fructose 1 reduced it further, to 82 (Figure 4C).

\section{Implications for Taste Processing}

These results contain interesting implications for taste coding. Taking first the question of total intensity, there was, as noted above, an apparent integration of the two sweetnesses. This integrated sweetness, in competition 

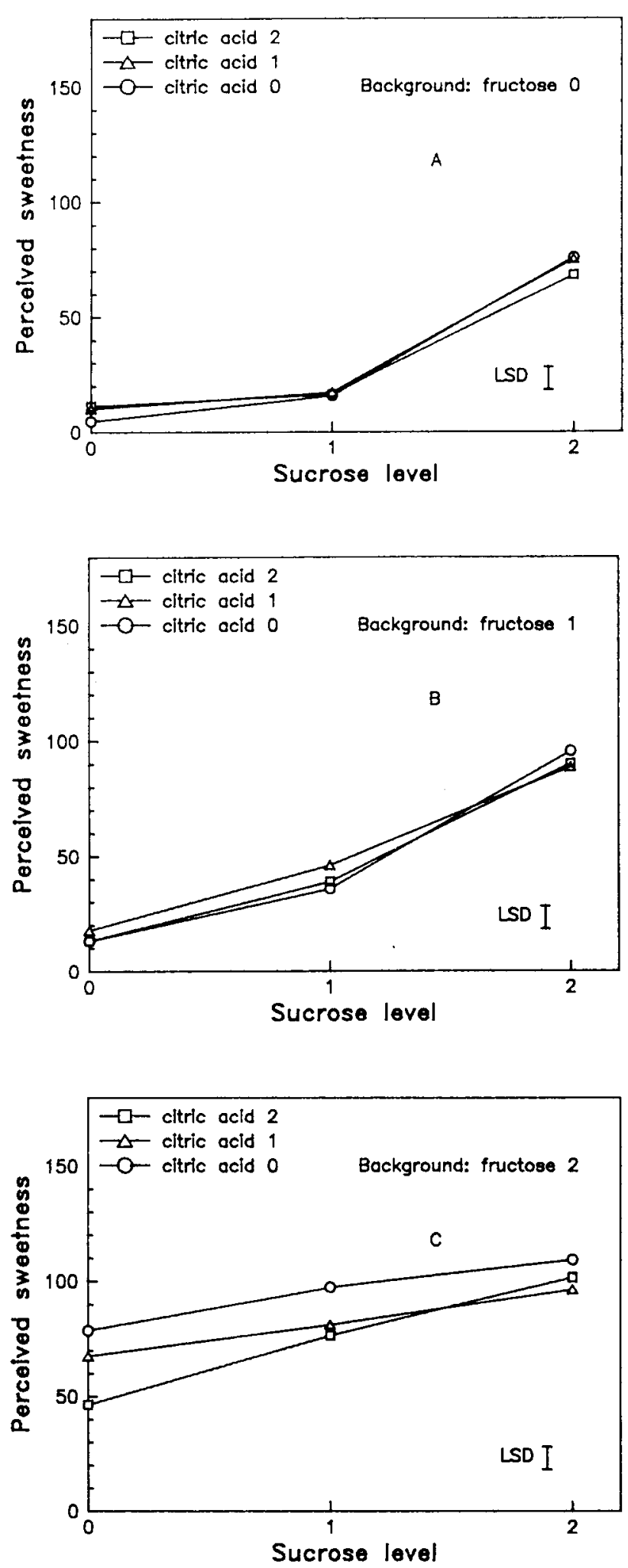

Figure 3. Mean sweetness ratings for the 27 sucrose/fructose/citric acid mixtures. Data presentation is as for Figure 1. with citric acid, dictates total intensity. But where does this integration occur? A recent experiment (McBride, 1988) has suggested that it is not a result of competition for binding at receptor sites; rather, it occurs somewhere "in" the taste system. This still leaves room for many possibilities, of course, although there is some indication (Jakinovich, 1982) that the integration might occur peripherally, in the taste effector system.

But the notion of peripheral integration is difficult to reconcile with the data on perception of the individual components, sweetness and acidity. If the sweetness integration were completed at a peripheral level, there would be only a single sweetness "signal" to interact with the citric acid "signal." However, as is shown in Figures 3 and 4, the sugars did not behave as one: When both were present in the mixture, citric acid selectively suppressed fructose more than sucrose; sucrose suppressed acidity more effectively than fructose. Had the two sweetnesses been irrevocably integrated, this selectivity could not have occurred.

There is another significant disparity between the processing of total intensity and processing of the individual components. As shown earlier in Figure 1, the total intensity of mixtures containing citric 2 was dictated by citric 2 alone; it was unaffected by the addition of sugars. Yet the acidity of citric 2 (Figure 4C) was profoundly affected by the addition of sugars: Presented alone, the acidity of citric 2 was rated at 120; when mixed with sucrose 2 /fructose 2 , the rating dropped to 76 . In other words, the total intensity of the acid component somehow reached consciousness unaffected by the sweetness, yet, at the same time, the actual taste quality of the acid component was markedly suppressed.

To digress briefly to the practical, this apparent contradiction may be an important, albeit unrecognized, principle in food formulation. Overall taste intensity- "impact" in the mouth-is important in the acceptability of food and drink (McBride \& Booth, 1986). Figure $4 \mathrm{im}-$ plies that a desirably high level of total intensity may be obtained in a product without the dominant component necessarily imparting all of its character to that product. For example, the acid component of a soft drink may simultaneously evoke high flavor intensity but only moderate acidity.

How might this effect be reconciled in terms of taste coding? One solution could be to distinguish between intensity coding and quality coding. Possibly, perceived intensity is determined at an early, peripheral stage. For homogeneous mixtures, this could mean integration in or before the effector system; for heterogeneous mixtures, it would mean no integration at all, with the more intense (dominant) component determining the intensity of the mixture. This hypothesis fits with the limited neurophysiological data on both homogeneous (Jakinovich, 1982) and heterogeneous (Smith, 1989) mixtures. Perception of taste quality, on the other hand, may be subject to further, cen- 

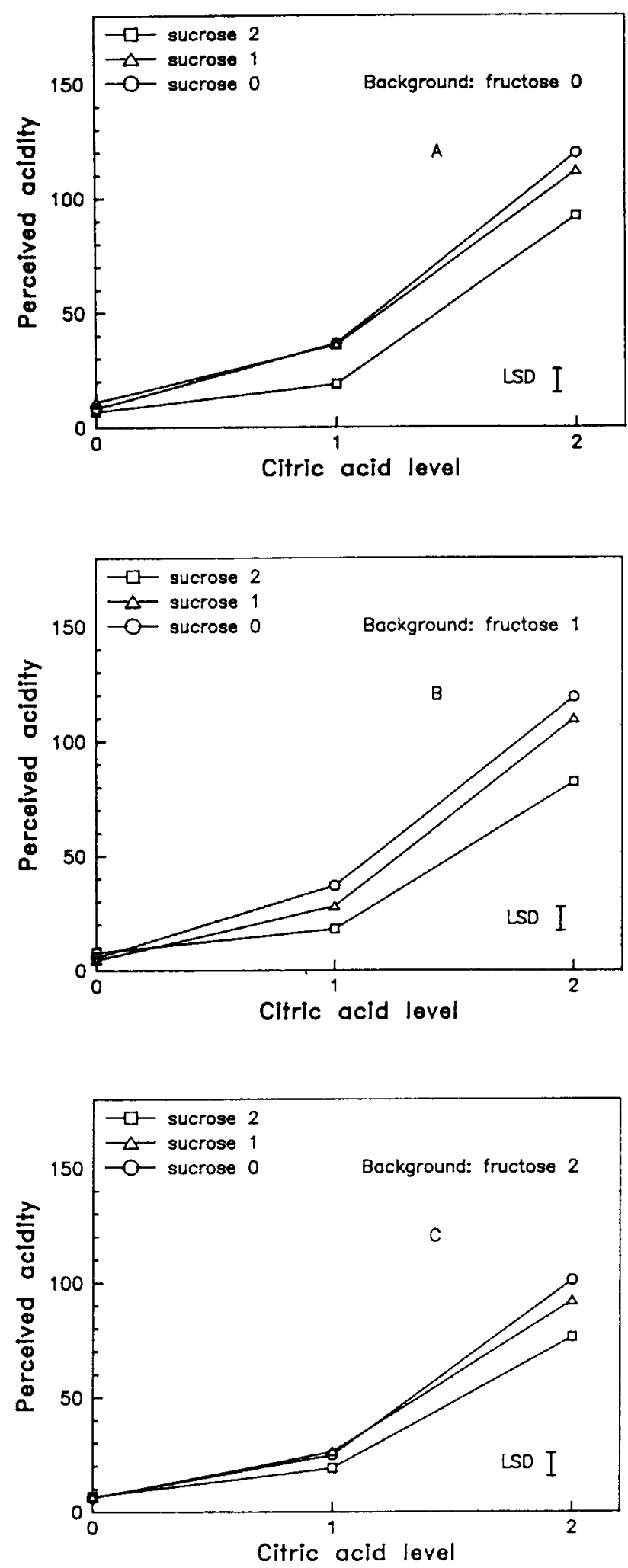

Figure 4. Mean acidity ratings of the 27 sucrose/fructose/citric acid mixtures. Citric acid concentration is given on the (log) abscissa, each curve represents a different level of sucrose, and each panel represents a different level of fructose. Within each panel, data points separated (vertically) by more than one $L S D$ are significantly difierent $(p<.05)$. tral coding. For heterogeneous mixtures, there are now indications that integration is a high-order, attentional phenomenon, occurring at a central level (Kroeze, 1982, 1989; Kroeze \& Bartoshuk, 1985; Lawless, 1979). A greater flexibility-readily practicable at the cognitive level-would seem to be necessary to accommodate the variety of effects in quality perception.

\section{REFERENCES}

De GraAf, C., \& Fruters, J. E. (1988). Assessment of the taste interaction between two qualitatively similar-tasting substances: A comparison between comparison rules. Journal of Experimental Psychology: Human Perception \& Performance, 14, 526-538.

Frank, R. A., \& ARChambo, G. (1986). Intensity and hedonic judgments of taste mixtures: An information integration analysis. Chemical Senses, 11, 427-438.

FrIJTERS, J. E. (1987). Psychophysical models for mixtures of tastants and mixtures of odorants. Annals of the New York Academy of Sciences, $510,67-78$.

JAKINOVICH, W., JR. (1982). Stimulation of the gerbil's gustatory receptors by saccharin. Journal of Neuroscience, 2, 49-56.

Kroeze, J. H. A. (1982). Mixture suppression in taste. Unpublished doctoral dissertation, University of Utrecht.

KroEZE, J. H. A. (1989). Is mixture suppression a peripheral or central event? In D. G. Laing, W. S. Cain, R. L. McBride, \& B. W. Ache (Eds.), Perception of complex smells and tastes (pp. 225-243). Sydney: Academic Press.

Kroeze, J. H. A., \& Bartoshuk, L. M. (1985). Bitterness suppression as revealed by split-tongue taste stimulation in humans. Physiology \& Behavior, 35, 779-783.

Laing, D. G., CAin, W. S., McBride, R. L., \& Ache, B. W. (Eds.) (1989). Perception of complex smells and tastes. Sydney: Academic Press.

LAWLESS, H. T. (1979). Evidence for neural inhibition in bittersweet taste mixtures. Joumal of Comparative \& Physiological Psychology, 93, 538-547.

MCBrude, R. L. (1982). Toward a unified theory of psychophysics. Unpublished doctoral dissertation, Macquarie University, Sydney.

MCBRIDE, R. L. (1986). Sweetness of binary mixtures of sucrose, fructose, and glucose. Journal of Experimental Psychology: Human Perception \& Performance, 12, 584-591.

MCBRIDE, R. L. (1987). Taste psychophysics and the Beidler equation. Chemical Senses, 12, 323-332.

MCBRIDE, R. L. (1988). Taste reception of binary sugar mixtures: Psychophysical comparison of two models. Perception \& Psychophysics, 44, 167-171.

MCBRIDE, R. L. (1989). Three models for taste mixtures. In D. G. Laing, W. S. Cain, R. L. McBride, \& B. W. Ache (Eds.), Perception of complex smells and tastes (pp. 265-282). Sydney: Academic Press.

MCBRIDE, R. L., \& ANDERSON, N. H. (in press). Integration psychophysics. In R. L. McBride \& H. J. H. MacFie (Eds.), Psychological basis of sensory evaluation. London: Elsevier.

MCBride, R. L., \& BOOTH, D. A. (1986). Using classical psychophysics to determine ideal flavour intensity. Journal of Food Technology, 21 , 775-780.

McBride, R. L., \& Finlay, D. C. (1989). Perception of taste mixtures by experienced and novice assessors. Journal of Sensory Studies, 3, 237-248.

MCBride, R. L., \& Johnson, R. L. (1987). Perception of sugar-acid mixtures in lemon juice drink. International Journal of Food Science and Technology, 22, 399-408.

SмITh, D. V. (1989). Neural and behavioral mechanisms of taste mixture perception in mammals. In D. G. Laing, W. S. Cain, R. L., McBride, \& B. W. Ache (Eds.), Perception of complex smells and tastes (pp. 149-170). Sydney: Academic Press.

(Manuscript received December 12, 1988; revision accepted for publication April 6, 1990.) 